\title{
Ipilimumab/Talimogene Laherparepvec Regimen
}

National Cancer Institute

\section{Source}

National Cancer Institute. Ipilimumab/Talimogene Laherparepvec Regimen. NCI

Thesaurus. Code C157051.

A chemotherapy regimen consisting of intralesional talimogene laherparepvec followed by intravenous ipilimumab that may be used in the treatment of melanoma. 